\title{
Oscillatory and steady streaming flow in the anterior chamber of the moving eye
}

\author{
M. Dvoriashyna ${ }^{1} \dagger$, R. Repetto ${ }^{1}$ and J. H. Tweedy ${ }^{2}$ \\ ${ }^{1}$ Department of Civil, Chemical and Environmental Engineering, University of Genoa, Via \\ Montallegro 1, Genoa, 16145, Italy \\ ${ }^{2}$ Department of Bioengineering, Imperial College London, London SW7 2AZ, UK
}

(Received $\mathrm{xx}$; revised $\mathrm{xx}$; accepted $\mathrm{xx}$ )

We study the flow induced by eye rotations in the anterior chamber (AC) of the eye, the region between the cornea and the iris. We model the geometry of the AC as a thin domain sitting on the surface of a sphere, and study both the simpler case of a constant-height domain as well as a more realistic AC shape. We model eye rotations as harmonic in time with prescribed frequency $\omega_{f}$ and amplitude $\beta$, and use lubrication theory to simplify the governing equations. We write the equations in a reference frame moving with the domain and show that fluid motion is governed by three dimensionless parameters: the aspect ratio of the $\mathrm{AC} \epsilon$, the amplitude $\beta$ and the Womersley number $\alpha$. We simplify the equations under the physiologically realistic assumptions that $\epsilon$ is small and $\alpha$ large, leading to a linear system that can be decomposed into three harmonics: a dominant frequency component, with frequency $\omega_{f}$, and a steady streaming component and a third component with frequency $2 \omega_{f}$. We solve the problem analytically for the constant-height domain and numerically as the solution of ODEs in the more realistic geometry. Both the primary flow and the steady streaming are shown to have a highly three-dimensional structure, which has not been highlighted in previous numerical works. We show that the steady streaming is particularly relevant from the clinical point of view, as it induces fluid mixing in the AC. Furthermore, the steady flow component is the dominant mixing mechanism during the night, when the thermal flow induced by temperature variations across the $\mathrm{AC}$ is suppressed.

\section{Introduction}

Pure frequency oscillations of a fluid are known to lead to a primary flow that oscillates with the same frequency as the driver, and secondary flows that are typically smaller and occur due to nonlinear forces, oscillating at integer multiples of the driving frequency. The steady streaming component is the contribution to the flow that has frequency zero. In a biological context the component oscillating at the driving frequency typically has primary influence on the stresses generated, since it is the largest component, while the steady streaming component is of interest since it has primary importance for mass transport.

In the present work we study the oscillatory and steady streaming flow in the anterior chamber of the eye (AC) generated during eye rotations. The bulk of the eye is filled with two main fluids: the vitreous and aqueous humours, see figure 1 . The rheology of the vitreous is that of a gel with both viscous and elastic properties that are thought to contribute to its dynamics (David et al. 1998; Meskauskas et al. 2011, 2012), while the aqueous is well characterised as a Newtonian fluid with rheological properties similar to those of

$\dagger$ Email address for correspondence: mariia.dvoriashyna@edu.unige.it 


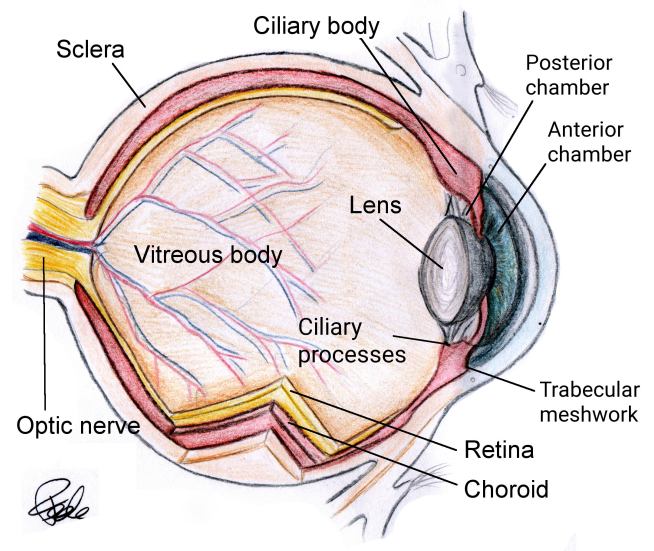

FIGURE 1. Sketch of a cross-section of the human eye.

water. Aqueous humour is produced by the ciliary processes at a rate of approximately $3 \mu \mathrm{l} / \mathrm{min}$ (Brubaker 1991) per eye, from where it flows in the posterior chamber, enters the AC through the pupil and is eventually drained through the trabecular meshwork and out of the eye.

In spite of being slow, aqueous flow has important functions, since the balance between the rate of production and resistance to drainage determines the intraocular pressure. This is particularly relevant clinically as an elevated intraocular pressure is correlated with the occurrence of open angle glaucoma (Sommer et al. 1991). The flow of aqueous humour is also important in the supply of nutrients to the avascular tissues of the lens and the cornea. Drugs placed on the surface of the eye are mainly drained into the AC, and their transport through the AC is strongly dependent on the flow of the aqueous (Urtti 2006). Furthermore, elevated shear stress on the cornea due to the flow of aqueous could lead to the detachment of endothelial cells from the cornea (Kaji et al. 2005).

Various mechanisms produce fluid flow in the AC of the eye, which have been fairly well studied from the mechanical point of view. The main mechanisms include: (i) aqueous secretion and drainage (Friedland 1978; Silver \& Quigley 2004; Fitt \& Gonzalez 2006; Villamarin et al. 2012; Repetto et al. 2015; Dvoriashyna et al. 2017); (ii) flow driven by buoyancy effects due to a temperature gradient across the AC (Canning et al. 2002; Heys \& Barocas 2002; Fitt \& Gonzalez 2006; Villamarin et al. 2012; Repetto et al. 2015); (iii) eye rotations (Abouali et al. 2012; Modarreszadeh et al. 2014; Repetto et al. 2015; Boushehrian et al. 2016); and (iv) Flow due to deformation of the shape of the anterior chamber, such as occurs during lens accommodation, accidents or if the eye is rubbed.

Repetto et al. (2015) showed that, of the first three mechanisms listed in the previous paragraph, rotations of the eye produce the most intense flow in the AC, and thus contribute to the majority of the wall shear stress on the cornea. Moreover, Abouali et al. (2012) showed that periodic rotations of the eye can produce a steady streaming flow that is intense enough to contribute to mixing at least as much as the thermally driven flow. When the eyes are closed during sleep, almost no thermal flow occurs in the $\mathrm{AC}$, meaning that most of the mixing happens due to the rapid eye movements (REM).

Analytical approaches based on lubrication theory have been extensively used to study the flow induced by aqueous secretion and the buoyancy driven flow in the AC, after the seminal work by Canning et al. (2002). On the other hand, all previous investigations of the flow induced by eye rotations are based on fully numerical solutions of the NavierStokes equations. Although numerical simulations allow one to consider complex and 
very realistic geometries, they require large computational efforts, especially for time dependent simulations, and make it difficult to obtain a clear picture of the dependency of the results on the controlling parameters. In this work we propose an analytical approach to study aqueous humour flow in the eye, which takes advantage of the small thickness of the AC, and use our results to investigate the parameter space more thoroughly than was previously possible.

Movements of the eye are routine and take a variety of different forms. Some rotations are close to periodic oscillations, and approximating them as a pure frequency oscillation has been a popular simplification in the literature, both in experimental works (Repetto et al. 2005; Bonfiglio et al. 2013, 2015) and theoretical works (David et al. 1998; Repetto et al. 2010; Abouali et al. 2012; Modarreszadeh et al. 2014). In this work we also make use of this assumption.

As long as the shape of the AC does not change during an eye movement, the motion can be decomposed into a translational motion and an instantaneous rotational motion about an axis. In the case of pure translational motion, there would be no fluid motion relative to the domain, and the acceleration is balanced by a pressure gradient within the fluid. In this paper we simplify the problem to the case of rotational motion, and, furthermore, we assume the axis of rotations remains fixed. Thus, in principle, our work can be generalised to any motion of the eye, by adding a pressure gradient onto our solutions.

We model the AC as a thin domain sitting on the surface of a sphere that either has a constant height (in which case we can solve the problem analytically) or a more realistic shape (in which case we reduce the problem to a set of ODEs). We investigate both the primary oscillatory flow and the steady streaming, showing that both have a threedimensional structure, the full details of which have not been highlighted by previous authors.

This paper is organised as follows: in section 2 the problem is formulated mathematically and simplified, and in section 3 the solution procedure is described. In section 4 we present the results, both in the constant height domain (section 4.1), and for the more realistic geometry (section 4.2 ). In section 5 we discuss the physiological and clinical relevance of the results.

\section{Formulation of the problem}

In this study we develop a model to find the motion of the aqueous humour during rotations of the eyeball. As discussed in the Introduction, the AC is relatively narrow in the anterior-posterior direction, and is delimited anteriorly by the cornea and posteriorly by the iris and lens, see figure 1 and the parameters given in table 1 . In this model, we describe the iris and lens as a single continuous surface, thus neglecting the passage of the aqueous through the iris-lens channel, and hence also the turnover of the aqueous due to secretion and drainage. We justify this by comparing the flows found in the present model with those that would be expected due to aqueous turnover (e.g. Repetto et al. 2015), and noting that the flows found in the present model are much larger.

\subsection{Geometry of the domain}

To describe the eye rotations and the shape of the domain, it is convenient to introduce a set of Cartesian coordinates and two sets of spherical polar coordinates, which will be used interchangeably through the paper, and which are all illustrated in figure 2.1. The $z$-axis of the Cartesian coordinates is the axis of rotation and the $x$-axis points through the centre of the pupil. The set $\mathcal{C}=\left(r^{*}, \theta, \phi\right)$ of spherical polar coordinates is convenient 


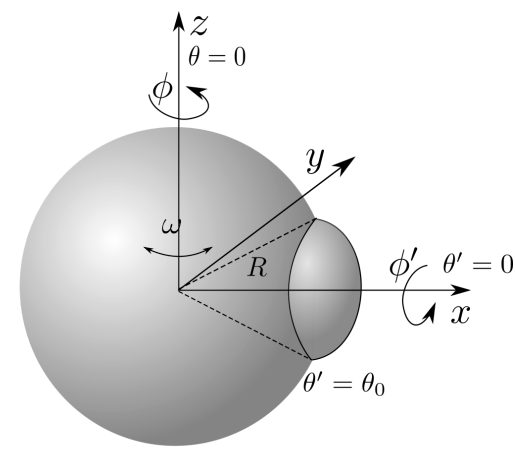

(a)

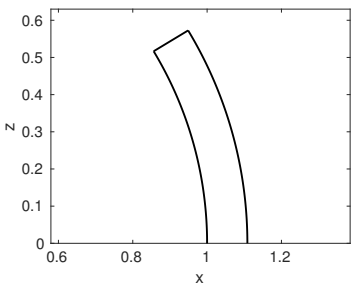

(b)

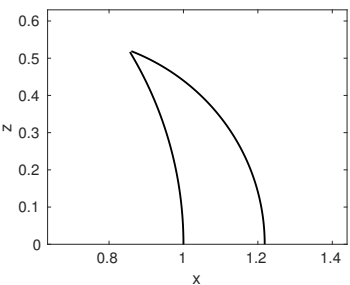

(c)

FiguRE 2. (a) Sketch of the eye, showing the eyeball (large sphere) and the domain representing the $\mathrm{AC}$ attached to the sphere on the right. Sketch of the coordinate systems $\mathcal{C}$ and $\mathcal{C}^{\prime}$. (b) and (c) cross-sections of the domains considered; (b) idealised constant-height domain; (c) more realistic representation of the $\mathrm{AC}$ geometry.

Maximum height, $h_{\max }$

Average height, $\bar{h}$

Diameter of the AC

Radius of the eyeball, $R$

Zenital boundary of the $\mathrm{AC}, \theta_{0}$

Aqueous humor kinematic viscosity, $\nu$

Aqueous humor density, $\rho$

$$
\begin{aligned}
& 2.62 \mathrm{~mm}(\text { ISO-11979-3 Helmholtz (1909)) } \\
& 1.3 \mathrm{~mm} \\
& \approx 6.2 \mathrm{~mm} \\
& \approx 12 \mathrm{~mm} \\
& 31.3^{\circ} \\
& 0.75 \cdot 10^{-6} \mathrm{~m}^{2} / \mathrm{s} \text { (Beswick \& McCulloch 1956) } \\
& 1000 \mathrm{~kg} / \mathrm{m}^{3}
\end{aligned}
$$

TABLE 1. Geometrical characteristics of the domain and fluid properties.

for describing the rotations, and has origin at the centre of the eye, $\theta=0$ along the axis of rotation and the centre of the pupil along the line $\theta=\pi / 2, \phi=0$. Finally the set $\mathcal{C}^{\prime}=\left(r^{*}, \theta^{\prime}, \phi^{\prime}\right)$ of spherical polar coordinates is convenient for describing the geometry of the $\mathrm{AC}$, and is obtained by rotating $\mathcal{C}$ through $-\pi / 2$ about the $y$-axis. Thus it has the same origin, with the line $\theta^{\prime}=0$ through the centre of the pupil and the axis of rotations along $\theta^{\prime}=\pi / 2, \phi^{\prime}=0, \pi$. The transformation from $\mathcal{C}$ to $\mathcal{C}^{\prime}$ can be performed using the following formulae (e.g. Meskauskas et al. 2011):

$$
\begin{array}{ll}
\cos \theta=-\sin \theta^{\prime} \cos \phi^{\prime}, & \cos \theta^{\prime}=\sin \theta \cos \phi, \\
\tan \phi=\tan \theta^{\prime} \sin \phi^{\prime}, & \tan \phi^{\prime}=-\tan \theta \sin \phi .
\end{array}
$$

To a good approximation, the $\mathrm{AC}$ is axisymmetric about the anterior-posterior axis $\left(\theta^{\prime}=0\right)$, and thus its geometry is independent of $\phi^{\prime}$. We also assume for simplicity that its posterior surface (the iris and the lens) is a region of a spherical surface of radius $R$ centred on the centre of the eye, see figure 2.1(a). In particular we neglect the thickness of the inner boundary of the iris at the iris-lens channel, which is about $0.4 \mathrm{~mm}$ in reality. In addition, the assumption that the centre of the posterior spherical surface and the centre of the eye coincide is not necessary (in reality the radius of curvature of the lens is around $1 \mathrm{~cm}$ and that of the eye is $1.2 \mathrm{~cm})$, but this allows us to find an analytical solution in the case of constant height. We denote the thickness of the AC as $h\left(\theta^{\prime}\right)$. We consider two cases: first, the case of uniform $h$, in which we set $h$ equal to the maximum height $h=h_{\max }$, see figure 2(b), which allows us to find a completely analytical solution for the fluid flow. Second, we consider we consider a more realistic shape in which the 
domain is defined as $R \leqslant r^{*} \leqslant R+h\left(\theta^{\prime}\right)$ for $0 \leqslant \theta^{\prime} \leqslant \theta_{0}$, where $h\left(\theta^{\prime}\right)$ is a smooth monotonic decreasing function with $h(0)=h_{\max }$. Since our method does not allow us to have $h$ reach zero at the outer boundary we arbitrarily set $h\left(\theta_{0}\right)$ to the small value $h_{\min }=h_{\max } / 50$. We adopted the following expression for $h$ :

$$
h=h_{\max } \cos \theta^{\prime}+\frac{h_{\min }-h_{\max } \cos \theta_{0}}{1-\cos \theta_{0}}\left(1-\cos \theta^{\prime}\right),
$$

which is illustrated in figure 2(c). We typically use the parameter values given in table 1 , except where otherwise stated, in which case the aspect ratio of the anterior chamber is $\epsilon=\bar{h} / R \approx 0.11$, where $\bar{h}$ is the average height of the AC (volume divided by area projected onto the plane $x=0$ ).

\subsection{Eye rotations}

In this work we consider harmonic oscillations of the domain, with amplitude $\beta$ and angular frequency $\omega_{f}$. Since we wish to apply our results to the flow in the AC during eye rotations, we adopt values representative of real eye movements and, in particular, of saccadic eye rotations and rapid eye movements (REM) during sleep.

Saccadic rotations are performed to redirect the sight from one target to another, and they tend to be fast and of short duration. Becker (1989) proposed empirical relationships relating saccadic amplitude $A$, duration $D$, maximum angular velocity and acceleration time. We relate $\beta$ and $\omega_{f}$ using the fact that for saccadic rotations $D=D_{0}+d A$, which is approximately valid in the range $5^{\circ} \lesssim A \lesssim 50^{\circ}$, with $D$ measured in seconds and $A$ in

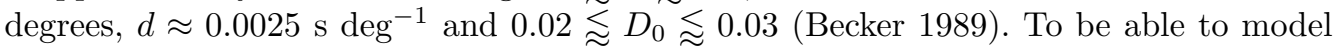
the eye rotations as saccadic movements, we assume that a period of rotation consists of two sucessive saccadic movements, each with amplitude $A$ and duration $D$, and thus model the saccade as a harmonic rotation with amplitude $\beta=A / 2$ and angular frequency $\omega_{f}=2 \pi /(2 D)=\pi /\left(D_{0}+2 d \beta\right)$. Note that this approach to model periodic saccades differs from the one in Abouali et al. (2012), where authors considered four saccadic movements per period instead of two. We nondimensionalise this relationship by converting $\beta$ to

radians and also working in terms of the Womersley number $\alpha=\sqrt{\rho \omega_{f} R^{2} / \mu}$, where $\rho$ is the fluid density and $\mu$ its shear viscosity (see table 1 ). This leads to the relationship shown by the curve in figure 3 .

REM is a normal feature of a particular phase of sleep, and consists of repeated side-to-side rotations that can have a wide range of amplitudes (Takahashi \& Atsumi 1997). For our model we adopt the same parameters as Modarreszadeh et al. (2014) and consider two cases: (i) 'average REM' with $\beta=6.27 \mathrm{deg}$ and $\omega_{f}=58.73 \mathrm{deg} / \mathrm{s}$, corresponding to the average of the experimental data by Takahashi \& Atsumi (1997), and (ii) 'maximum amplitude REM' with $\beta=61.5 \mathrm{deg}$ and $\omega_{f}=491.98 \mathrm{deg} / \mathrm{s}$, which is the highest amplitude reported in the measurements, and these points are also reported in figure 3.

\subsection{Derivation of the governing equations}

In the following we work in a reference frame that rotates with the domain, and using superscript stars to denote dimensional variables that will be made dimensionless. Neglecting gravity, the continuity and Navier-Stokes equations become (e.g. Batchelor 


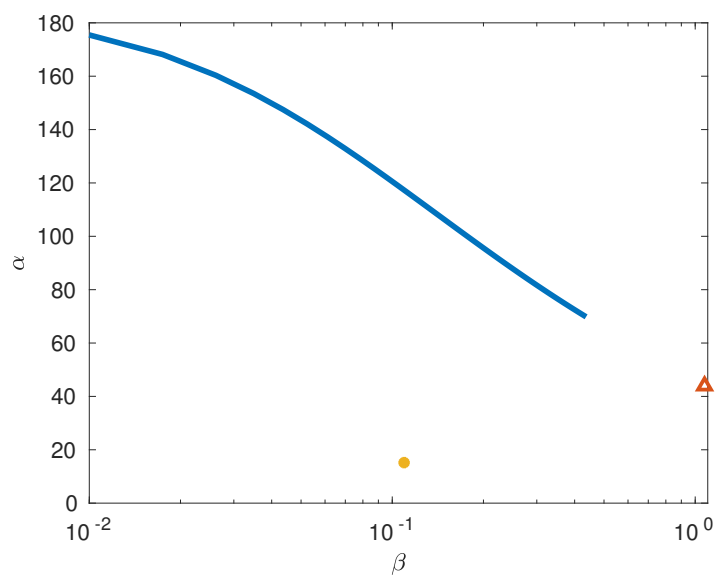

FiguRE 3. Relationship between the Womersley number $\alpha$ and the amplitude of rotations $\beta$ for saccadic eye rotations (curve), average REM (star) and maximum amplitude REM (triangle) (Modarreszadeh et al. 2014).

1967)

$$
\begin{aligned}
& \nabla^{*} \cdot \mathbf{u}^{*}=0, \\
& \rho\left(\dot{\mathbf{u}}^{*}+\left(\mathbf{u}^{*} \cdot \nabla^{*}\right) \mathbf{u}^{*}+\dot{\boldsymbol{\omega}}^{*} \times \mathbf{x}^{*}+2 \boldsymbol{\omega}^{*} \times \mathbf{u}^{*}+\left(\boldsymbol{\omega}^{*} \cdot \mathbf{x}^{*}\right) \boldsymbol{\omega}^{*}-\omega^{* 2} \mathbf{x}^{*}\right)= \\
& -\nabla^{*} p^{*}+\mu \nabla^{2} \mathbf{u}^{*}
\end{aligned}
$$

where $\mathbf{u}^{*}$ is fluid velocity relative to the reference frame, $p^{*}$ is the departure of the pressure from the hydrostatic profile (thus we omit the gravitational acceleration), $\boldsymbol{\omega}^{*}$ is the prescribed angular velocity of the domain (and $\omega^{*}=\left|\boldsymbol{\omega}^{*}\right|$ ), $\mathbf{x}^{*}$ is the position vector, $\boldsymbol{\nabla}^{*}$ is spatial gradient and a dot denotes a dimensional time derivative. Recalling the utilised coordinate system $\mathcal{C}=\left(r^{*}, \theta, \phi\right)$, we consider sinusoidal rotations with prescribed angular displacement $\beta \sin \omega_{f} t^{*}$ about the axis $\theta=0(z=0)$, giving the angular velocity $\boldsymbol{\omega}^{*}=\omega^{*}(\cos \theta,-\sin \theta, 0)$ with magnitude $\omega^{*}=\beta \omega_{f} \cos \omega_{f} t^{*}$.

A characteristic depth of the anterior chamber may be obtained by calculating the volume of the anterior chamber divided by inner surface area, which, assuming $h \ll R$, is approximately $\bar{h}=\int_{0}^{\theta_{0}} h\left(\theta^{\prime}\right) \sin \left(\theta^{\prime}\right) d \theta^{\prime} /\left(1-\cos \left(\theta_{0}\right)\right)$. Dividing by the length of the chamber gives the aspect ratio of the anterior chamber $\epsilon=\bar{h} /\left(2 R \theta_{0}\right)$.

We introduce the following scales for nondimensionalisation:

$$
\begin{gathered}
r^{*}=R+\bar{h} r, \quad t^{*}=\frac{t}{\omega_{f}}, \quad u_{\phi}^{*}=U u_{\phi}, \quad u_{\theta}^{*}=U u_{\theta}, \quad u_{r}^{*}=\epsilon U u_{r} \\
p^{*}=P_{0} p, \quad \omega^{*}=\beta \omega_{f} \omega .
\end{gathered}
$$

We choose $U=\bar{h} \beta \omega_{f}$ as the velocity scale and $P_{0}=R^{2} \omega_{f}^{2} \beta \rho$ as the pressure scale (chosen so that the pressure gradient in the $\phi$ direction balances the angular acceleration term), and let $\epsilon=\bar{h} / R$ be the approximate aspect ratio of the domain.

With the above scales the dimensionless continuity and Navier-Stokes equations (2.3) 
Type

Small saccades

Large saccades

Average REM

Maximum amplitude REM

$\begin{array}{ccccccc}A & \beta & \omega_{f}, 1 / \mathrm{s} & \alpha & \epsilon \beta & 1 / \alpha^{2} & 1 / \epsilon \alpha^{2} \\ 5^{\circ} & 0.04 & 96.7 & 136.2 & 0.0048 & 5.4 \cdot 10^{-5} & 4.8 \cdot 10^{-4} \\ 50^{\circ} & 0.43 & 21.7 & 64.5 & 0.049 & 2.4 \cdot 10^{-4} & 2.1 \cdot 10^{-3} \\ 12.54^{\circ} & 0.11 & 1.03 & 14 & 0.012 & 5.1 \cdot 10^{-3} & 4.6 \cdot 10^{-2} \\ 123^{\circ} & 1.07 & 8.58 & 40.6 & 0.12 & 6.1 \cdot 10^{-4} & 5.4 \cdot 10^{-3}\end{array}$

TABLE 2. Typical values of the dimensionless parameters, with $\epsilon \approx 0.11$ in all cases.

read

$$
\begin{aligned}
& \frac{\partial u_{r}}{\partial r}+\frac{1}{\sin \theta} \frac{\partial}{\partial \theta}\left(u_{\theta} \sin \theta\right)+\frac{1}{\sin \theta} \frac{\partial u_{\phi}}{\partial \phi}+\epsilon\left(r \frac{\partial u_{r}}{\partial r}+2 u_{r}\right)=0 \\
& \epsilon^{3} \frac{\partial u_{r}}{\partial t}+\epsilon^{4} \beta \mathbf{u} \cdot \nabla u_{r}-\epsilon^{3} \beta \frac{u_{\phi}^{2}+u_{\theta}^{2}}{1+\epsilon r}-2 \beta \epsilon^{2} \omega u_{\phi} \sin \theta-\epsilon \beta \omega^{2}(1+\epsilon r) \sin ^{2} \theta= \\
& \quad=-\frac{\partial p}{\partial r}+\frac{\epsilon}{\alpha^{2}}\left(\nabla^{2} u_{r}-\epsilon^{2} \frac{2 u_{r}}{(1+\epsilon r)^{2}}-\frac{2 \epsilon}{(1+\epsilon r)^{2} \sin \theta}\left(\frac{\partial\left(u_{\theta} \sin \theta\right)}{\partial \theta}+\epsilon \frac{\partial u_{\phi}}{\partial \phi}\right)\right),
\end{aligned}
$$

$$
\begin{gathered}
\epsilon \frac{\partial u_{\theta}}{\partial t}+\epsilon^{2} \beta \mathbf{u} \cdot \nabla u_{\theta}+\epsilon^{3} \beta \frac{u_{r} u_{\theta}}{1+\epsilon r}-\epsilon^{2} \beta \frac{u_{\phi}^{2} \cot \theta}{1+\epsilon r}-2 \epsilon \beta \omega \cos \theta u_{\phi}-\beta \omega^{2}(1+\epsilon r) \cos \theta \sin \theta= \\
=-\frac{1}{1+\epsilon r} \frac{\partial p}{\partial \theta}+\frac{1}{\epsilon \alpha^{2}}\left(\nabla^{2} u_{\theta}+\frac{2 \epsilon^{3}}{(1+\epsilon r)^{2}} \frac{\partial u_{r}}{\partial \theta}-\frac{\epsilon^{2}}{r^{2} \sin ^{2} \theta}\left(u_{\theta}+2 \cos \theta \frac{\partial u_{\phi}}{\partial \phi}\right)\right)
\end{gathered}
$$

$$
\begin{gathered}
\epsilon \frac{\partial u_{\phi}}{\partial t}+\epsilon^{2} \beta \mathbf{u} \cdot \nabla u_{\phi}+\epsilon^{3} \beta \frac{u_{\phi} u_{r}}{r}+\epsilon^{2} \beta \frac{u_{\theta} u_{\phi} \cot \theta}{r}+(1+\epsilon r) \dot{\omega} \sin \theta+ \\
+2 \epsilon \beta \omega\left(u_{\theta} \cos \theta+\epsilon u_{r} \sin \theta\right)=-\frac{1}{(1+\epsilon r) \sin \theta} \frac{\partial p}{\partial \phi}+
\end{gathered}
$$$$
+\frac{1}{\epsilon \alpha^{2}}\left(\nabla^{2} u_{\phi}+\frac{2 \epsilon^{2}}{(1+\epsilon r)^{2} \sin \theta}\left(\frac{\partial u_{r}}{\partial \phi}+\cot \theta \frac{\partial u_{\phi}}{\partial \phi}\right)-\epsilon^{3} \frac{u_{\phi}}{(1+\epsilon r)^{2} \sin ^{2} \theta}\right)
$$

with $\omega=\cos t$ and $\dot{\omega}=-\sin t$, and

$$
\begin{aligned}
\nabla^{2} f & =\frac{\partial^{2} f}{\partial r^{2}}+\frac{2 \epsilon}{1+\epsilon r} \frac{\partial f}{\partial r}+\frac{\epsilon^{2}}{(1+\epsilon r)^{2} \sin \theta} \frac{\partial}{\partial \theta}\left(\sin \theta \frac{\partial f}{\partial \theta}\right)+\frac{\epsilon^{2}}{\sin ^{2} \theta(1+\epsilon r)^{2}} \frac{\partial^{2} f}{\partial \phi^{2}}= \\
& =\frac{\partial^{2} f}{\partial r^{2}}+2 \epsilon \frac{\partial f}{\partial r}+\mathcal{O}\left(\epsilon^{2}\right),
\end{aligned}
$$

for any function $f$.

Using the geometrical values from table 1 , we estimate

$$
\epsilon \approx 0.11, \quad \epsilon^{2} \approx 0.012
$$

and in table 2 we report the remaining dimensionless parameters appearing in the governing equations (2.5) in four cases (taken from the data in figure 3): small and large saccadic rotations (amplitude $5^{\circ}$ and $50^{\circ}$, respectively), average REM and maximum amplitude REM.

In the remainder of this work we drop from equations (2.5) any terms of orders $\epsilon^{2}, \epsilon \beta$, $1 / \alpha^{2}$, which can be seen to be small from table 2 , as well as smaller terms. This leaves terms of order $1, \beta, \epsilon$ and $1 /\left(\epsilon \alpha^{2}\right)$. Despite the fact that $1 /\left(\epsilon \alpha^{2}\right)$ is typically smaller than both $\epsilon^{2}$ and $\epsilon \beta$ we choose to keep terms with this coefficient, as they are not negligible within the boundary layer. The same approach was used by Blondeaux \& Vittori (1994) 
for a different problem, who showed that it leads to the same results as formal boundary layer analysis. Note also, that for maximum amplitude REM, terms of order $\epsilon \beta$ are comparable to those of order $\epsilon$, implying that the model might not be very accurate in this case.

With the above simplifications we obtain the following system:

$$
\begin{aligned}
& \frac{\partial p}{\partial r}=0, \\
& \epsilon \frac{\partial u_{\theta}}{\partial t}-\beta \omega^{2} \cos \theta \sin \theta=-(1-\epsilon r) \frac{\partial p}{\partial \theta}+\frac{1}{\epsilon \alpha^{2}} \frac{\partial^{2} u_{\theta}}{\partial r^{2}}, \\
& \epsilon \frac{\partial u_{\phi}}{\partial t}+(1+\epsilon r) \dot{\omega} \sin \theta=-\frac{1-\epsilon r}{\sin \theta} \frac{\partial p}{\partial \phi}+\frac{1}{\epsilon \alpha^{2}} \frac{\partial^{2} u_{\phi}}{\partial r^{2}},
\end{aligned}
$$

coupled to the continuity equation (2.5a), which remains in full. Equations (2.8) should be solved subject to no-slip boundary conditions at $r=0$ and $r=h(\theta, \phi)$ and zero flux at $\theta^{\prime}=\theta_{0}$.

\section{Solution}

\subsection{Decomposition of the solution}

As is usual in lubrication theory, equation (2.8a) shows that the pressure does not depend on the $r$-coordinate. The motion is forced by the term proportional to $\omega^{2}$ in equation $(2.8 b)$ and that proportional to $\dot{\omega}$ in equation $(2.8 c)$. Recalling that $\omega=\cos t=$ $e^{\mathrm{i} t} / 2+$ c.c., where c.c. denotes the complex conjugate, we note that three frequencies are effectively forced: 0,1 and 2 , corresponding to the terms proportional to $e^{0 \mathrm{it} t}, e^{\mathrm{i} t}$ and $e^{2 i t}$. In other words, harmonic oscillations of the domain with dimensionless frequency 1 , force a steady streaming component, a flow with the same frequency as the forcing and also flow at twice that frequency. Thus, we seek a solution for the velocity and the pressure in the form

$$
\begin{aligned}
& \mathbf{u}(\mathbf{x}, t)=\mathbf{u}^{(0)}(\mathbf{x})+\mathbf{u}^{(1)}(\mathbf{x}) e^{\mathrm{i} t}+\mathbf{u}^{(2)}(\mathbf{x}) e^{2 \mathrm{i} t}+\text { c.c. }, \\
& p(\mathbf{x}, t)=p^{(0)}(\mathbf{x})+p^{(1)}(\mathbf{x}) e^{\mathrm{i} t}+p^{(2)}(\mathbf{x}) e^{2 i t}+\text { c.c. }
\end{aligned}
$$

Substituting into equations $(2.8 a)-(2.8 c)$ we obtain the following set of equations for $k=0,1,2$, respectively:

$$
\begin{aligned}
& \frac{\partial p^{(k)}}{\partial r}=0 \\
& \mathrm{i} k \epsilon u_{\theta}^{(k)}-\frac{1}{\epsilon \alpha^{2}} \frac{\partial^{2} u_{\theta}^{(k)}}{\partial r^{2}}=-(1-\epsilon r) \frac{\partial p^{(k)}}{\partial \theta}+\Theta^{(k)}, \\
& \mathrm{i} k \epsilon u_{\phi}^{(k)}-\frac{1}{\epsilon \alpha^{2}} \frac{\partial^{2} u_{\phi}^{(k)}}{\partial r^{2}}=-\frac{1-\epsilon r}{\sin \theta} \frac{\partial p^{(k)}}{\partial \phi}+\Phi^{(k)},
\end{aligned}
$$

where $\Theta^{(0)}=\Theta^{(2)}=(\beta / 8) \sin (2 \theta), \Phi^{(1)}=-(\mathrm{i} / 2)(1+\epsilon r) \sin \theta$ and $\Theta^{(1)}=\Phi^{(0)}=\Phi^{(2)}=$ 0 . Equations (3.3) are coupled with the continuity equation for each harmonic.

\subsection{Derivation of the equation for the pressure}

Following the standard approach in lubrication theory, we solve equations $(3.3 b)$ and (3.3c) to find the $r$-dependence of $u_{\theta}^{(k)}$ and $u_{\phi}^{(k)}$, respectively, which, when combined with the no-slip boundary conditions gives

$$
\mathbf{u}_{\mathrm{h}}^{(k)}=A_{k}(r, h) \nabla_{\mathrm{h}} p^{(k)}+B_{k}(r, h) \mathbf{v}_{k},
$$


for $k=0,1,2$, where $\mathbf{v}_{0}=\mathbf{v}_{2}=\sin 2 \theta \mathbf{e}_{\theta}, \mathbf{v}_{1}=\sin \theta \mathbf{e}_{\phi}$ and the functions $A_{k}$ and $B_{k}$ are reported in the appendix. The unit vectors $\mathbf{e}_{\theta}$ and $\mathbf{e}_{\phi}$ point in the directions of increasing $\theta$ and $\phi$, respectively and a subscript $\mathrm{h}$ on a vector indicates that the projection of the vector on the $\theta$ and $\phi$ directions is taken.

It is convenient from now on to work in the coordinate system $\mathcal{C}^{\prime}$ defined in Section 2.1, so that we can make use of the fact that the domain is axisymmetric with respect to $\theta^{\prime}=0$ (see figure 2.1). In this coordinate system (3.4) takes the form

$$
\mathbf{u}_{\mathrm{h}}^{(k)}=A_{k}(r, h) \nabla_{\mathrm{h}}^{\prime} p^{(k)}+B_{k}(r, h) \mathbf{v}_{k}^{\prime},
$$

where $\mathbf{v}_{0}^{\prime}=\mathbf{v}_{2}^{\prime}=-\sin 2 \theta^{\prime} \cos ^{2} \phi^{\prime} \mathbf{e}_{\theta^{\prime}}+\sin 2 \phi^{\prime} \sin \theta^{\prime} \mathbf{e}_{\phi^{\prime}}, \mathbf{v}_{1}^{\prime}=\sin \phi^{\prime} \mathbf{e}_{\theta^{\prime}}+\cos \theta^{\prime} \cos \phi^{\prime} \mathbf{e}_{\phi^{\prime}}$ and $\nabla^{\prime}$ is the gradient operator in the $\mathcal{C}^{\prime}$ coordinate system. Integrating the continuity equation $(2.5 a)$ over $r$, we obtain a partial differential equation for $p^{(k)}$ for $k=0,1,2$ :

$$
\mathcal{A}\left(\gamma_{k}\right) p^{(k)}+\mathcal{B}_{k}\left(\delta_{k}\right)=0
$$

where

$$
\gamma_{k}(h)=\int_{0}^{h}(1+\epsilon r) A_{k}(r, h) \mathrm{d} r, \quad \delta_{k}(h)=\int_{0}^{h}(1+\epsilon r) B_{k}(r, h) \mathrm{d} r,
$$

(explicit expressions for $\gamma_{k}$ and $\delta_{k}$ appear in the appendix), and $\mathcal{A}\left(\gamma_{k}\right)$ is a linear differential operator given by

$$
\mathcal{A}\left(\gamma_{k}\right)=\frac{1}{\sin \theta^{\prime}}\left(\frac{\partial}{\partial \theta^{\prime}}\left(\sin \theta^{\prime} \gamma_{k} \frac{\partial}{\partial \theta^{\prime}}\right)+\frac{\gamma_{k}}{\sin \theta^{\prime}} \frac{\partial^{2}}{\partial \phi^{\prime 2}}\right)
$$

with

$$
\begin{aligned}
& \mathcal{B}_{0}\left(\delta_{0}\right)=2\left(3 \sin ^{2} \theta^{\prime} \cos ^{2} \phi^{\prime}-1\right) \delta_{0}(h)-2 \cos ^{2} \phi^{\prime} \sin \theta^{\prime} \cos \theta^{\prime} \frac{\mathrm{d} \delta_{0}(h)}{\mathrm{d} \theta^{\prime}} \\
& \mathcal{B}_{1}\left(\delta_{1}\right)=\sin \phi^{\prime} \frac{\partial \delta_{1}}{\partial \theta^{\prime}} \\
& \mathcal{B}_{2}\left(\delta_{2}\right)=\mathcal{B}_{0}\left(\delta_{2}\right)
\end{aligned}
$$

The $r$-integrated velocity, or flux per unit length, reads

$$
\mathbf{q}_{\mathrm{h}}^{(k)}=\int_{0}^{h}(1+\epsilon r) \mathbf{u}_{\mathrm{h}}^{(k)} \mathrm{d} r=\gamma_{k} \nabla_{\mathrm{h}}^{\prime} p^{(k)}+\delta_{k} \mathbf{v}_{k}^{\prime}
$$

where $\nabla_{\mathrm{h}}^{\prime}$ is computed on the spherical surface $r=1$.

The no-flux condition at the boundary $\theta^{\prime}=\theta_{0}$ can then be written as

$$
\mathbf{q}_{\mathrm{h}}^{(k)} \cdot \mathbf{e}_{\theta^{\prime}}=0 \Rightarrow \gamma_{k} \frac{\partial p^{(k)}}{\partial \theta^{\prime}}+\delta_{k} \mathbf{v}_{k}^{\prime} \cdot \mathbf{e}_{\theta^{\prime}}=0
$$

and we also require the solution to be regular at $\theta^{\prime}=0$, meaning that $p^{(k)}$ is finite there.

In the following subsections, we simplify the problem for each harmonic $k=0,1,2$ by separating the $\theta^{\prime}$ - and $\phi^{\prime}$-dependence, and we derive an analytical solution for each in the case of constant $h$. Note that each $p^{(k)}$ is uniquely defined up to the addition of an arbitrary constant. 


\subsection{Separation of variables and solution for the case of constant $h$}

\subsubsection{Dominant frequency component $(k=1)$}

For the case $k=1$ we note the $\phi^{\prime}$-dependence in (3.6) and (3.11) and seek a solution of the form

$$
p^{(1)}\left(\theta^{\prime}, \phi^{\prime}\right)=g\left(\theta^{\prime}\right) \sin \phi^{\prime}
$$

This leads to

$$
\frac{1}{\sin \theta^{\prime}} \frac{\mathrm{d}}{\mathrm{d} \theta^{\prime}}\left(\sin \theta^{\prime} \gamma_{1} \frac{\mathrm{d} g}{\mathrm{~d} \theta^{\prime}}\right)-\frac{\gamma_{1}}{\sin ^{2} \theta^{\prime}} g+\frac{\mathrm{d} \delta_{1}}{\mathrm{~d} \theta^{\prime}}=0
$$

with boundary condition

$$
\frac{\mathrm{d} g}{\mathrm{~d} \theta^{\prime}}=-\frac{\delta_{1}}{\gamma_{1}} \quad \text { at } \theta^{\prime}=\theta_{0} .
$$

In the general case (3.13) must be solved numerically, but in the special case of constant $h, \gamma_{1}$ and $\delta_{1}$ are both constant, and we obtain

$$
\begin{aligned}
& p^{(1)}=-\frac{\delta_{1}}{\gamma_{1}} \frac{\sin ^{2} \theta_{0}}{1-\cos \theta_{0}} \frac{1-\cos \theta^{\prime}}{\sin \theta^{\prime}} \sin \phi^{\prime}, \\
& u_{r}^{(1)}=0, \\
& u_{\theta}^{(1)}=\left(B_{1}(r)-A_{1}(r) \frac{\delta_{1}}{\gamma_{1}} \frac{\sin ^{2} \theta_{0}}{1-\cos \theta_{0}} \frac{1-\cos \theta^{\prime}}{\sin ^{2} \theta^{\prime}}\right) \sin \phi^{\prime}, \\
& u_{\phi}^{(1)}=\left(B_{1}(r) \cos \theta^{\prime}-A_{1}(r) \frac{\delta_{1}}{\gamma_{1}} \frac{\sin ^{2} \theta_{0}}{1-\cos \theta_{0}} \frac{1-\cos \theta^{\prime}}{\sin ^{2} \theta^{\prime}}\right) \cos \phi^{\prime},
\end{aligned}
$$

where we have set the arbitrary constant of integration to zero in the expression for $p^{(1)}$, and $u_{r}^{(1)}$ was found from the continuity equation. The expressions for the $r$-integrated velocity is reported in the Appendix.

\subsubsection{Steady streaming $(k=0)$ and double frequency $(k=2)$ components}

For the cases $k=0$ and $k=2$ the procedure is similar, and we derive the solutions together in this section. Noting the $\phi^{\prime}$-dependence in (3.6) and (3.11), we seek a solution of the form

$$
p^{(k)}\left(\theta^{\prime}, \phi^{\prime}\right)=f_{0}^{(k)}\left(\theta^{\prime}\right)+f_{1}^{(k)}\left(\theta^{\prime}\right) \cos 2 \phi^{\prime},
$$

which leads to

$$
\begin{gathered}
\frac{1}{\sin \theta^{\prime}} \frac{\mathrm{d}}{\mathrm{d} \theta^{\prime}}\left(\gamma_{k} \sin \theta^{\prime} \frac{\mathrm{d} f_{0}^{(k)}}{\mathrm{d} \theta^{\prime}}\right)-\sin \theta^{\prime} \cos \theta^{\prime} \frac{\mathrm{d} \delta_{k}}{\mathrm{~d} \theta^{\prime}}+\left(3 \sin ^{2} \theta^{\prime}-2\right) \delta_{k}=0, \\
\frac{1}{\sin \theta^{\prime}} \frac{\mathrm{d}}{\mathrm{d} \theta^{\prime}}\left(\gamma_{k} \sin \theta^{\prime} \frac{\mathrm{d} f_{1}^{(k)}}{\mathrm{d} \theta^{\prime}}\right)-\frac{4 \gamma_{k} f_{1}^{(k)}}{\sin ^{2} \theta^{\prime}}-\sin \theta^{\prime} \cos \theta^{\prime} \frac{\mathrm{d} \delta_{k}}{\mathrm{~d} \theta^{\prime}}+3 \sin ^{2} \theta^{\prime} \delta_{k}=0 .
\end{gathered}
$$

with boundary conditions

$$
\frac{\mathrm{d} f_{0,1}^{(k)}}{\mathrm{d} \theta^{\prime}}=\frac{\delta_{k}}{2 \gamma_{k}} \sin 2 \theta^{\prime} \quad \text { at } \theta^{\prime}=\theta_{0} .
$$

As for the harmonic $k=1$, in the general case (3.16) must be solved numerically, but 
in the case of constant $h$ we obtain the following analytical solution

$$
\begin{aligned}
& p^{(k)}=-\frac{\delta_{k}}{2 \gamma_{k}}\left(1-2 \cos ^{2} \phi^{\prime} \sin ^{2} \theta^{\prime}\right), \\
& u_{r}^{(k)}=-\left(\frac{\delta_{k} \hat{\gamma}_{k}(r)}{\gamma_{k}}-\hat{\delta}_{k}(r)\right) \frac{\left(1+3 \cos 2 \theta^{\prime}\right) \cos ^{2} \phi^{\prime}-2 \cos 2 \phi^{\prime}}{(1+\epsilon r)^{2}}, \\
& u_{\theta}^{(k)}=\left(A_{k}(r) \frac{\delta_{k}}{\gamma_{k}}-B_{k}(r)\right) \sin 2 \theta^{\prime} \cos ^{2} \phi^{\prime}, \\
& u_{\phi}^{(k)}=-\left(A_{k}(r) \frac{\delta_{k}}{\gamma_{k}}-B_{k}(r)\right) \sin 2 \phi^{\prime} \sin \theta^{\prime},
\end{aligned}
$$

where we have set the arbitrary constant to zero in the expression for $p^{(k)}, u_{r}^{(k)}$ was found from the continuity equation, and $\hat{\gamma}_{k}(r)=\int_{0}^{r}(1+\epsilon s) A_{k}(s) \mathrm{d} s$ and $\hat{\delta}_{k}=\int_{0}^{r}(1+\epsilon s) B_{k}(s) \mathrm{d} s$. It is straightforward to check that the $r$-integrated velocity $\mathbf{q}_{h}^{(k)}$ is zero for $k=0,2$.

\section{Results}

\subsection{Constant-height domain}

We start with the special case of constant $h$. In figure 4 we show the flow component with frequency $1(k=1)$, which we expect to be much larger than the other two components $(k=0,2)$. We show the flow and pressure via snapshots at four different times on the different rows. The first row $(t=0)$ corresponds to the time when the angular velocity is maximum and the domain is moving in the direction of the positive $y$-axis, and the subsequent rows correspond to subsequent times spaced $1 / 8$ th period apart.

In the first column of plots in figure 4 , we show the velocity on the horizontal midplane $z=0$ and $y>0$. In each case the fluid close to the inner wall moves in the same direction as the rotation and that near the outer wall moves in the opposite direction (with the exception of $t=\pi / 2$ when the domain is not moving, figure $4(\mathrm{~g}))$. Note that the fact that the velocity does not go to zero at the boundary $\theta^{\prime}=\theta_{0}$ (as can be seen in the first and second column) is because, due to the simplification of the lubrication theory, we can only impose zero flux there rather than a full no-slip condition. Close to $\theta^{\prime}=\theta_{0}$ the assumptions of lubrication theory do not hold, and the radial velocity component would be comparable to the other components.

In the second column of figure 4, we show the $r$-integrated velocity field obtained from equation (3.10) and the pressure distribution on the surface $r=1$, both projected onto a plane of constant $x$. At $t=0$ the angular acceleration is zero, and thus the pressure is almost constant on the surface $r=0$, figure $4(\mathrm{~b})$, but the pressure gradient is much larger at the other three times, especially at $t=\pi / 2$ when the angular acceleration peaks, figure 4(h). Each plot shows two circulations that are reflections of one another, which indicates that the instantaneous flow has a highly three-dimensional structure.

The third column of figure 4 shows the velocity component $u_{\theta^{\prime}}$ along the line $\theta^{\prime}=0$, which is a region of the positive $x$-axis with $x=1+\epsilon r$. Each line corresponds to a different value of the Womersley number $\alpha$. Inspection of the plots shows that, as the Womersley number increases, a progressively thinner oscillatory Stokes boundary layer forms at both walls, as expected.

In figure 5 we show the steady streaming flow on the vertical plane $y=0$. We only plot the upper half of the domain since the flow is symmetric in the $x$-axis, as can be seen by inspecting equations $(3.17 b)$ and $(3.17 c)$. In the outer part of the domain fluid 


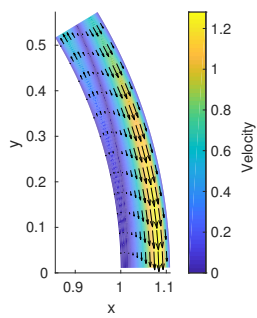

(a)

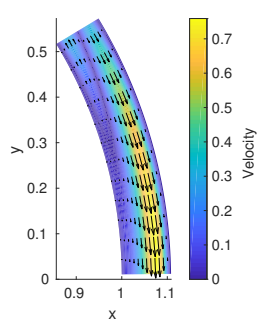

(d)

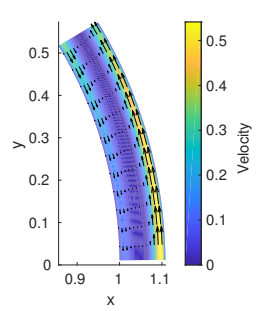

(g)

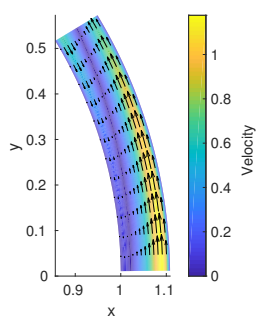

(j)

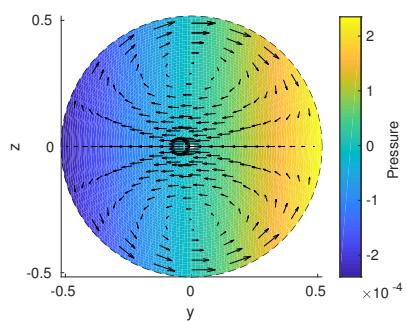

(b)

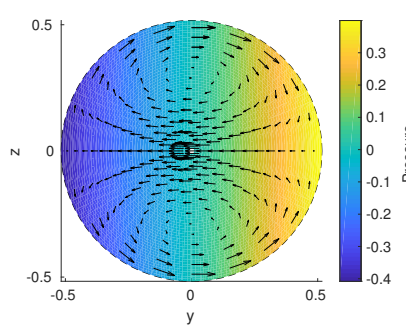

(e)

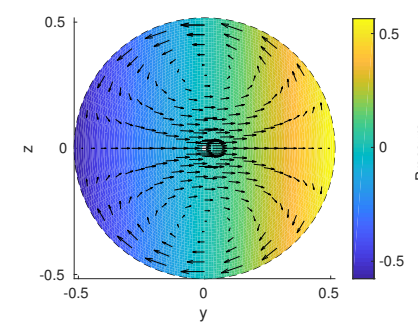

(h)

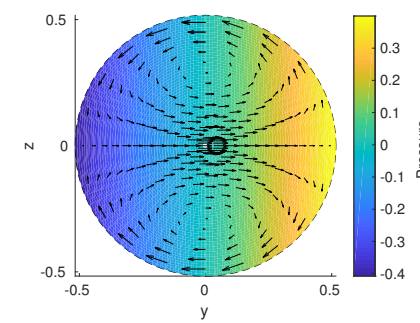

$(\mathrm{k})$

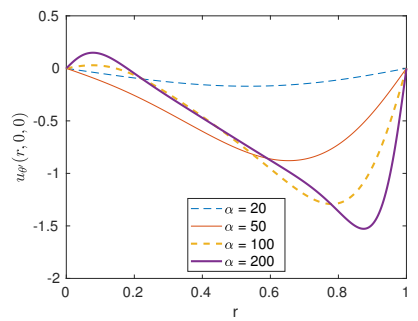

(c)

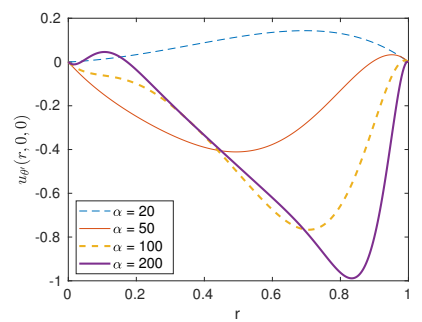

(f)

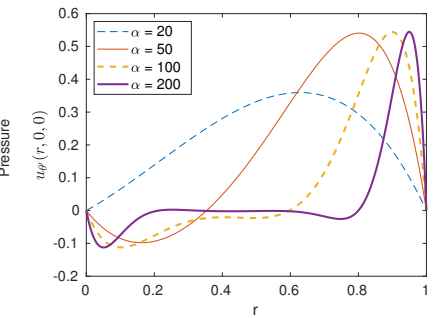

(i)

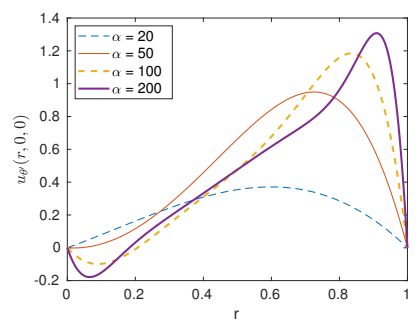

(l)

Figure 4 . The flow of the dominant harmonic component $(k=1)$ in the special case $h$ constant: The four rows show four equally spaced times through the first half period (those during the second half period are the reflections of these): $t=0, \pi / 4, \pi / 2$ and $3 / 4 \pi$, respectively. First column: arrows indicate fluid velocity relative to the domain $\mathbf{u}^{(1)}$ and shading indicates its dimensionless magnitude on half of the midplane $z=0$ (the other half is an antisymmetric reflection). Second column: dimensionless pressure $p^{(1)}$ at $r=0$ (shading) and $r$-integrated velocity $\mathbf{q}_{\mathrm{h}}^{(\mathbf{1})}$ (arrows), projected onto a plane of constant $x$. Third column: profiles of the relative velocity component $u_{\theta^{\prime}}$ along the axis $\theta^{\prime}=0$. For all plots we used $\beta=6.5^{\circ}$ and $\epsilon=0.11$, and in the first two columns we used $\alpha=100$. The velocity vectors in the first two columns are normalised so that their maximum length is the same in all plots. 


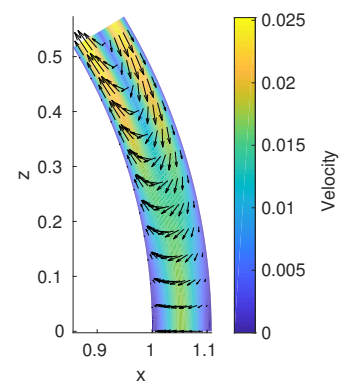

FiguRE 5. The steady streaming flow in the special case of a domain of constant height, shown on half of the midplane $y=0\left(\phi^{\prime}=\pi\right)$ (the other half is a symmetric reflection). The arrows indicate the relative velocity $\mathbf{u}^{(0)}$ and colours indicate its magnitude. The parameter values are $\alpha=100, \beta=6.5^{\circ}, \epsilon=0.11$.

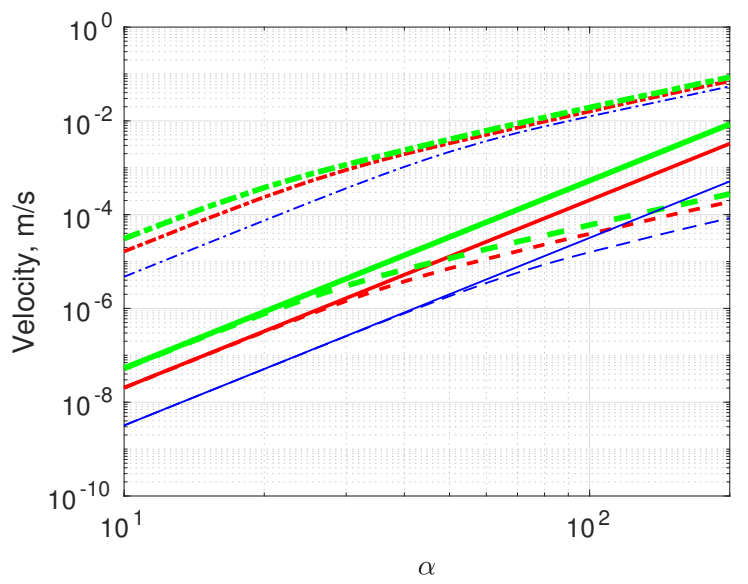

FiguRE 6. Velocity magnitudes over a range of frequencies in the special case of a constant height domain. Maximum value (over both time and space) attained by the magnitude of the velocity as a function of the Womersley number $\alpha$. The different line styles denote the different harmonics: $k=0$ (solid); $k=1$ (dot-dashed); and $k=2$ (dashed); and the thickness and color show the values of $\epsilon: \epsilon \approx 0.15$ (thick green); $\epsilon \approx 0.11$ (medium red); and $\epsilon \approx 0.06$ (thin blue). The amplitude is fixed at $\beta=6.5^{\circ}$ in each case.

particles move towards the midplane $z=0$ and in the inner part of the domain they move away from $z=0$. This is consistent with what is found in a periodically rotating sphere (Repetto et al. 2008; Colombini 2014), where a circulation with the same sense of rotation is found, and also with the numerical findings of Abouali et al. (2012), relative to the flow in the AC. Since the model does not capture the behaviour of the oscillatory flow close to the boundary of the domain (at $\theta^{\prime}=\theta_{0}$ ), we cannot rule out the possibility that boundary effects could produce a steady streaming in the core of the domain. We recall that in the case of the constant thickness domain the $r$-integrated velocity is equal to zero, and so we do not show it.

In figure 6 we show the maximum dimensional value over time and space attained by the velocity for each of the harmonics as a function of the Womersley number $\alpha$. As expected the magnitude of the dominant harmonic $k=1$ velocity is much larger than the others. Moreover, the steady streaming velocity has a significantly larger magnitude than the harmonic $k=2$ for large values of $\alpha$. 


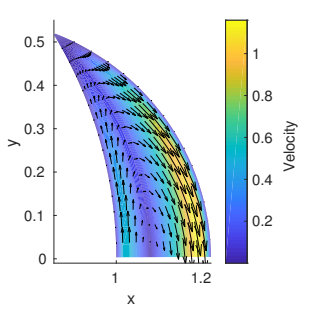

(a)

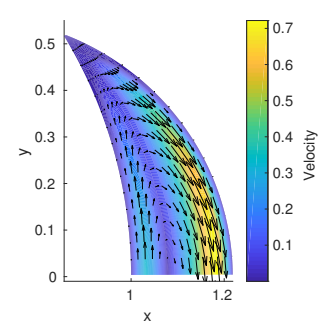

(d)

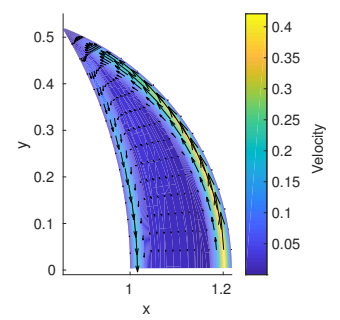

(g)

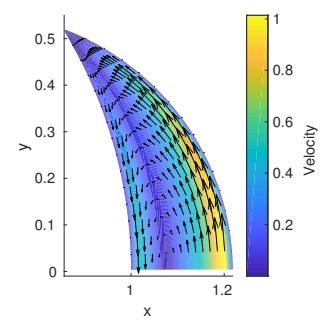

(j)

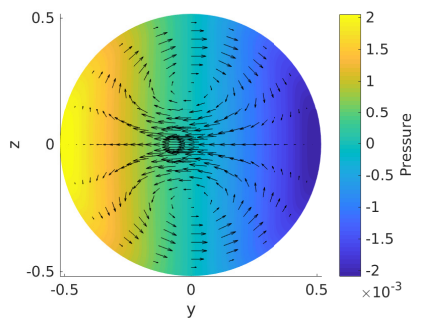

(b)

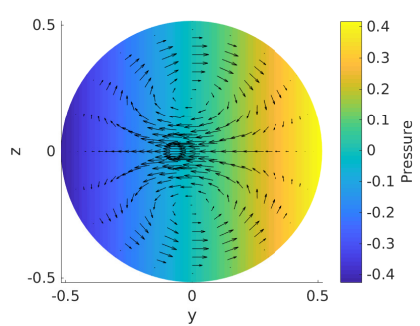

(e)

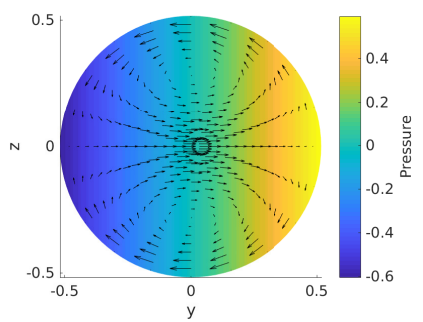

(h)

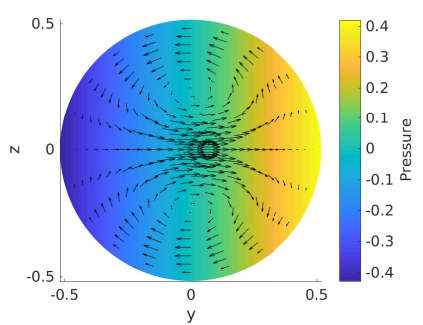

(k)

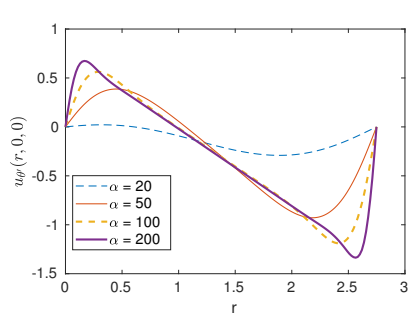

(c)

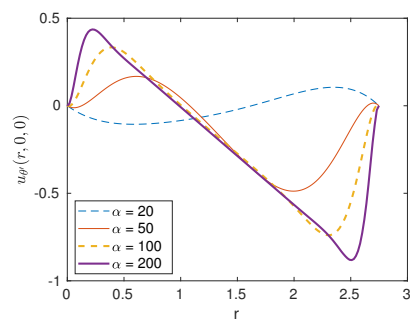

(f)

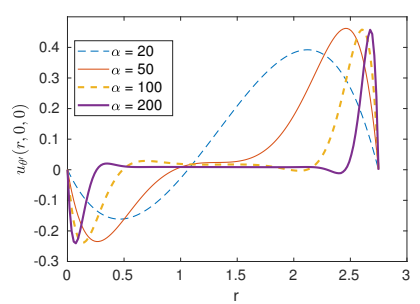

(i)

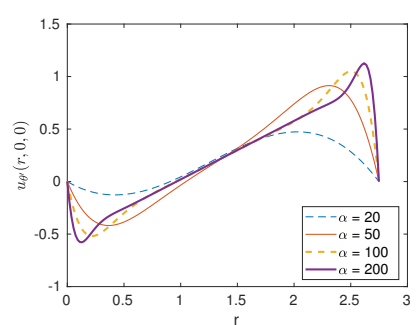

(l)

Figure 7. As for figure 4, but for the case of the more realistic eye shape described in the introduction of Section 2.1.

\subsection{More realistic eye geometry}

In this section we report the results obtained using the more realistic shape of the anterior chamber $(\mathrm{AC})$ of the eye, shown in figure 2(c). Figure 7 is the analogy of figure 4 for this case, and mostly shows qualitatively similar findings. However, in the case of the more realistic domain shape, the velocity decreases near the periphery of the domain $\left(\theta^{\prime}\right.$ near to $\left.\theta_{0}\right)$, since the domain is thinner there. It is interesting to note that the circulations 


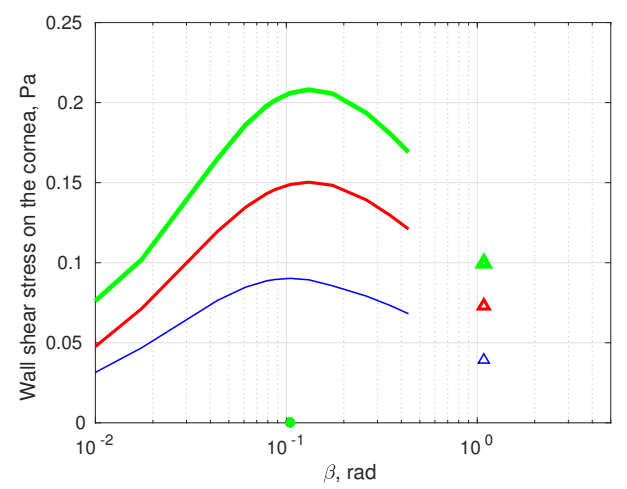

Figure 8. Maximum dimensional value over space and time of the wall shear stress on the cornea as a function of the angular amplitude of the saccadic eye rotation $\beta$ in the case of the more realistic eye shape. For each value of $\beta$ the curve for saccades in figure 3 was used to find the corresponding value of $\alpha$. The lines with different thickness and color correspond to different values of $h_{\max }$ : medium red: $h_{\max }=2.62 \mathrm{~mm}(\epsilon \approx 0.11)$; thin blue: $h_{\max }=1.5 \mathrm{~mm}$ $(\epsilon \approx 0.06)$; thick green: $h_{\max }=3.5 \mathrm{~mm}(\epsilon \approx 0.15)$. The symbols refer to REM: triangles represent maximum amplitude REM and stars average REM, with their thickness and color corresponding to maximum height of the domain considered. The three symbols for average REM cannot be distinguished as they are approximately on top of one another.

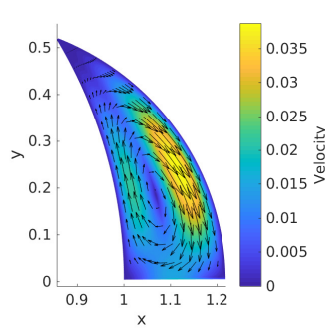

(a)

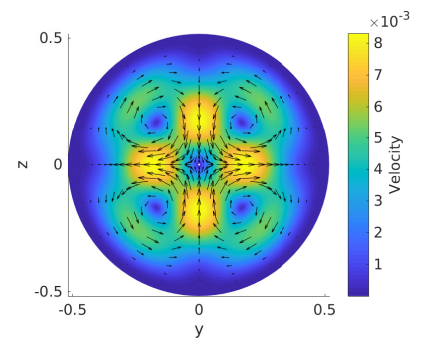

(b)

FiguRE 9. The steady streaming flow for the more realistic domain shape: (a) Relative velocity $\mathbf{u}^{(0)}$ on the vertical cross section $y=0$; (b) The $r$-integrated velocity, projected onto the $(y, z)$-plane. In both figures, colours indicate velocity magnitude and arrows show magnitude and direction. The parameters are $\alpha=100, \beta=6.5^{\circ}, \epsilon=0.11$.

in the $r$-integrated velocity fields that were observed in the case of the constant height domain are still present in this case, suggesting that the flow induced by eye rotations is highly three-dimensional.

In figure 8 we plot the maximum dimensional value over space and time of the wall shear stress on the cornea as a function of the eye rotation amplitude in the case of both saccades and REM. We compared the results for three different values of maximum thickness of the AC, within the physiological range (e.g. Jivrajka et al. 2008). The maximum wall shear stress increases with the height of the domain, and for a given domain, peaks for saccade amplitudes around $0.15 \mathrm{rad}$ or $8.6^{\circ}$.

Figure 9 shows the steady streaming, which indicates similar behaviour to the constantheight case, figure 5, but in this case a closed circulation is visible in figure $9(\mathrm{a})$, which forms since the domain gets thin close to the periphery $\theta^{\prime}=\theta_{0}$. Furthermore, the $r$ integrated velocity is non-zero, unlike in the constant-height case, meaning that the streaming flow is fully three-dimensional. In this case this flow is characterised by four 


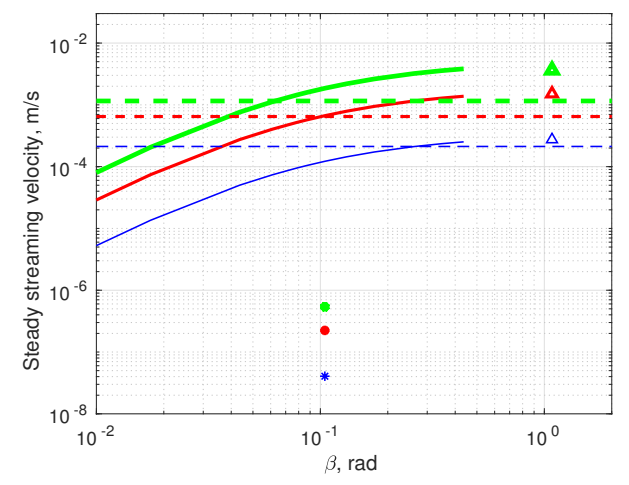

FiguRE 10. Maximum over space and time of the magnitude of the steady streaming flow as a function of the angular amplitude of the eye rotation. The solid curves and symbols are as in figure 8 . The thermal flow due to body heating is also shown (as horizontal dashed lines since they do not correspond to a particular value of $\beta$ ), colored and having width representing thickness of the domain (as in figure 8).

symmetrically arranged circulations, which are shown in figure 9 (b). Their magnitude is smaller than that on the midplane shown in figure $9(\mathrm{a})$, but they are likely to have physiological importance, as they lead to transverse mixing in the AC.

Figure 10 shows the dependence of the maximum dimensional steady streaming velocity on the saccade amplitude, with the colours corresponding to different thicknesses of the domain. Maximum steady streaming velocity increases with the saccade amplitude. The steady streaming velocity of maximum amplitude REM is also similar, whereas that of average REM is much lower. Increasing the thickness of the AC leads to higher steady streaming velocities in each case.

\section{Discussion}

We have studied the flow in a thin domain performing harmonic rotations about a fixed axis, in order to investigate the flow in the anterior chamber of the eye (AC) induced by eye rotations. As mentioned in the Introduction, this is relevant to the study of nutrient and drug delivery to the tissues of the eye, and allows us to estimate the shear stresses on the cornea, which could lead to the detachment of endothelial cells.

We worked in a reference frame moving with the domain, and assumed that the aspect ratio of the AC is small, the Womersley number $\alpha$ is large and the angular amplitude $\beta<1 \mathrm{rad}$, which allowed us to reduce the governing equations to a linear system forced by the angular and the centrifugal accelerations. We considered both the case of a domain with constant thickness, for which we found a fully analytical solution, and a more realistic approximation of the AC geometry. The flow has three components: the largest oscillates with the forcing frequency, and the others are a steady streaming component and a component that has twice the fundamental frequency.

In both the cases of a constant height chamber and the more realistic shape, we find that the dominant frequency component of the flow has a complex three-dimensional structure, which has not been observed in previous numerical simulations of the flow in the AC (Abouali et al. 2012; Modarreszadeh et al. 2014). This is because in our analysis we work in a frame moving with the domain, making some of the details of the flow easier to observe, since they are not disguised by the velocities of the chamber itself. The dominant frequency component of the flow is associated with much higher 


$\begin{array}{lllll} & \text { Saccade } 6^{\circ} & \text { Saccade } 30^{\circ} & \text { average REM } & \text { max amplitude REM } \\ \text { This work } & 0.094 & 0.1 & 0.2 \cdot 10^{-3} & 0.074 \\ \text { Previous works } & 0.12 & 0.147 & 0.248 \cdot 10^{-3} & 0.06\end{array}$

TABle 3. Comparison of the maximum WSS (in $\mathrm{Pa}$ ) on the cornea calculated with the present model with the results from numerical works of Abouali et al. (2012) (saccades) and Modarreszadeh et al. (2014) (REM).

velocities than the other components, and therefore contributes the majority of the wall shear stress (WSS) on the cornea. The WSS is larger for higher Womersley numbers as boundary layers develop, and it is also larger for thicker chambers. We found that WSS is maximised in the centre of the cornea (see also Abouali et al. 2012), and reaches a maximum value of around $0.15 \mathrm{~Pa}$ for $\beta \approx 8.6^{\circ}$ and $\alpha=124$. A direct comparison of our results with those of Abouali et al. (2012) is not possible, since we model the saccades differently: in that work, four saccades per period were used, whereas in our model we use two. We therefore adapted our model to use the same saccadic motion, and present the results in Table 3, which show good agreement between our model and the work of both Abouali et al. (2012) and Modarreszadeh et al. (2014). The WSS values predicted by both our model and the models of Abouali et al. (2012) and also Repetto et al. (2015) are of a similar order of magnitude to those found to cause detachment $(\sim 0.1 \mathrm{~Pa})$ by Kaji et al. (2005), who studied the corneal endothelial cells in vitro. This suggests that higher values of WSS would be required in vivo for the corneal cells to detach. Note that the values of the WSS due to eye rotations are much larger than those produced by the boyancy driven flow due to temperature differences in the AC, which is about $0.0016 \mathrm{~Pa}$ (e.g Repetto et al. 2015).

The steady streaming flow is forced by the centrifugal acceleration, and its velocity is much smaller than that of the flow oscillating with the forcing frequency, but nevertheless can have a key role in mixing processes, as it is time independent. The steady streaming flow has a complicated three-dimensional structure, which was not fully highlighted in the numerical simulations of Abouali et al. (2012) and Modarreszadeh et al. (2014), and has a non zero $r$-integrated velocity. We note that Modarreszadeh et al. (2014) also observed smaller vortices close to the pupil boundary and next to the trabecular meshwork, which we cannot capture with our model due to our geometrical simplifications and use of lubrication theory.

In order to estimate the effective importance of the steady streaming flow on mixing in the $\mathrm{AC}$, we compute the Péclet number, which measures the strength of advection over diffusion, and is given by $\mathrm{Pe}=L U / D$, where $D$ is the diffusion coefficient, $L$ is the radius of the $\mathrm{AC}$ and $U$ is characteristic velocity. With $D=10^{-9} \mathrm{~m}^{2} / \mathrm{s}$ (Lide 1996), $L=6.2 \mathrm{~mm}$ and a typical value of the velocity of $5 \cdot 10^{-4} \mathrm{~m} / \mathrm{s}$ (see figure 10 ) we obtain $\mathrm{Pe} \approx \mathcal{O}\left(10^{3}\right)$. The $r$-integrated steady streaming is much smaller, and the corresponding Péclet number is of order $10^{2}$. In both cases it is clear that advection largely dominates over diffusion, and confirms that eye rotations have a key role in governing transport processes in the AC.

Besides eye rotations, the other mechanism that is known to produce a flow that significantly contributes to fluid mixing is the temperature gradient across the AC, since it also leads to the generation of a steady flow. To compare the steady streaming flow during saccades with the thermal flow we make use of the results of Canning et al. (2002), who developed an idealised model in which the geometry of the AC was approximated as a spherical cap to derive an analytical expression for the thermal flow. This showed 
that the flow is directed along the vertical planes. The maximum magnitude of the fluid velocity was found to be

$$
u_{T, \max }=\frac{\Delta T \rho g \alpha_{T} h_{\max }^{2} \sqrt{3}}{216 \nu},
$$

where $\Delta T$ is the temperature difference between the cornea and the iris, $\alpha_{T}=3 \cdot 10^{-4} \mathrm{~K}^{-1}$ is the coefficient of thermal expansion, $g$ is gravitational acceleration, $\rho$ and $\nu$ are fluid density and kinematic viscosity, respectively and $h_{\max }$ is the maximum thickness of the anterior chamber (see table 1 ). With $\Delta T=3 \mathrm{~K}$, we estimate $u_{T, \max }=6.47 \cdot 10^{-4} \mathrm{~m} / \mathrm{s}$. Tweedy et al. (2017) used a similar method and, using their code with the same geometry as used here, they found a maximum velocity of $6.84 \cdot 10^{-4} \mathrm{~m} / \mathrm{s}$, while Repetto et al. (2015) used a numerical method for a slightly different geometry and found a maximum velocity of $5.92 \cdot 10^{-4} \mathrm{~m} / \mathrm{s}$ for $\Delta T=3 \mathrm{~K}$, which are both close to the value derived analytically from (5.1). We therefore assume that (5.1) provides a sufficiently accurate estimate for our purposes. We compare the thermal flow and the steady streaming flow induced by eye rotations in figure 10, which show that the steady flow induced by eye rotations has the same order of magnitude for large saccades and REM. Thus the two flows both effectively contribute to mixing in the AC.

During the night, thermally driven flow becomes insignificant, and, aside from shape changes of the $\mathrm{AC}$, the mechanisms driving flow are the REM and aqueous secretion. The maximum magnitude of the latter is $\approx 5.89 \cdot 10^{-5} \mathrm{~m} / \mathrm{s}$ (Repetto et al. 2015) at the pupil and its average velocity is $\approx 8 \cdot 10^{-6} \mathrm{~m} / \mathrm{s}$, which are both significantly smaller than the steady streaming flow found in the work. This provides confirming evidence for the hypothesis of Maurice (1998), which is that REM could promote mixing in the otherwise almost stagnant aqueous humour in order to maintain the nutrient supply to the cornea.

This work neglects any interactions between the flow induced by eye rotations and the other flow mechanisms in the AC. For small fluid velocities, due to linearity, the total flow may be approximated as the sum of the flow due to saccades, that due to secretion and drainage of the aqueous and the thermally driven flow. However, any flow due to shape deformations in the $\mathrm{AC}$ would be fully interactive with the other flows and would thus require a separate study.

To summarise, we find good agreement with previous numerical solutions and highlight characterestics of the flow that were not previously described. We show that the flow induced by eye rotations is a significant part of the aqueous humour dynamics and an important player in mixing processes. Finally, we remark that the model developed here could be useful for other applications involving oscillating thin domains.

\section{Acknowledgements}

This work was financially supported by Ophtec BV (The Netherlands). The authors thank Prof. Federica Grillo for drawing figure 1 and Paolo Soleri for many useful suggestions.

\section{Appendix A. Definition of the functions introduced in section 3}

We report functions appearing in the expressions used in section 3. 
Oscillatory and steady streaming flow in the anterior chamber of the moving eye 19

A.1. Harmonic $k=0$

$$
\begin{aligned}
& A_{0}(r, h)=\frac{\epsilon \alpha^{2}}{2}\left(r(r-h)-\frac{\epsilon r}{3}\left(r^{2}-h^{2}\right)\right), \\
& B_{0}(r, h)=\frac{\beta \epsilon \alpha^{2}}{16}\left(r h-r^{2}\right), \\
& \gamma_{0}(h)=\int_{0}^{h}(1+\epsilon r) A_{0}(r, h) d r=\frac{\beta \epsilon \alpha^{2} h^{3}}{180 \beta}\left(4 \epsilon^{2} h^{2}-15\right), \\
& \delta_{0}(h)=\int_{0}^{h}(1+\epsilon r) B_{0}(r, h) d r=\frac{\beta \epsilon \alpha^{2}}{96}\left(\frac{\epsilon h^{4}}{2}+h^{3}\right) .
\end{aligned}
$$

\section{A.2. Harmonic $k=1$}

$$
\begin{aligned}
& A_{1}(r, h)=\frac{i}{\epsilon}\left(\frac{\sin a r(\epsilon h+\cos a h-1)}{\sin a h}-\cos a r-(\epsilon r-1)\right) \\
& B_{1}(r, h)=\frac{1}{2 \epsilon}\left(\frac{\sin a r(\epsilon h-\cos a h+1)}{\sin a h}+\cos a r-(\epsilon r+1)\right) \\
& \gamma_{1}(h)=-i\left(\frac{\epsilon h^{3}}{3}-\frac{h}{\epsilon}-\frac{\epsilon h}{a^{2}}+\frac{2-2 \cos a h+\epsilon^{2} h^{2} \cos a h}{\epsilon a \sin a h}\right) \\
& \delta_{1}(h)=-\frac{1}{2}\left(\frac{\epsilon h^{3}}{3}+h^{2}+\frac{h}{\epsilon}-\frac{h \epsilon}{a^{2}}+\frac{\epsilon\left(\epsilon h^{2}+2 h\right) \cos a h-2 \epsilon h+2(\cos a h-1)}{\epsilon a \sin a h}\right)
\end{aligned}
$$

where $a=\sqrt{-i} \epsilon \alpha$.

The components of the $r$-integrated velocity are

$$
\begin{aligned}
& q_{\theta}^{(1)}=\delta_{1}(r)\left(1-\frac{\sin ^{2} \theta_{0}}{1-\cos \theta_{0}} \frac{1-\cos \theta^{\prime}}{\sin ^{2} \theta^{\prime}}\right) \sin \phi^{\prime}, \\
& q_{\phi}^{(1)}=\delta_{1}(r)\left(\cos \theta^{\prime}-\frac{\sin ^{2} \theta_{0}}{1-\cos \theta_{0}} \frac{1-\cos \theta^{\prime}}{\sin ^{2} \theta^{\prime}}\right) \cos \phi^{\prime} .
\end{aligned}
$$

A.3. Harmonic $k=2$

$$
\begin{aligned}
A_{2}(r, h) & =\frac{i}{2 \epsilon}\left(\frac{\sin b r(\epsilon h+\cos b h-1)}{\sin b h}-\cos b r-(\epsilon r-1)\right), \\
\gamma_{2}(r, h) & =-\frac{i}{2}\left(\frac{\epsilon h^{3}}{3}-\frac{h}{\epsilon}-\frac{\epsilon h}{b^{2}}+\frac{2-2 \cos b h+\epsilon^{2} h^{2} \cos b h}{\epsilon b \sin b h}\right), \\
B_{2}(r, h) & =\frac{i \beta}{16 \epsilon}\left(\sin b r \frac{1-\cos b h}{\sin b h}+\cos b r-1\right), \\
\delta_{2}(r, h) & =-\frac{i \beta}{16 \epsilon}\left(\frac{(2+\epsilon h)(\cos b h-1)}{b \sin b h}+h+\frac{\epsilon h^{2}}{2}\right) .
\end{aligned}
$$

where $b=\sqrt{-2 i} \epsilon \alpha$.

\section{REFERENCES}

Abouali, O., Modareszadeh, A., Ghaffarieh, A. \& Tu, J. 2012 Investigation of saccadic eye 
movement effects on the fluid dynamic in the anterior chamber. Journal of biomechanical engineering 134 (2), 021002, PMID: 22482669.

Batchelor, G. K. 1967 An Introduction to Fluid Dynamics. Cambridge University Press.

BeckeR, W. 1989 Metrics. In The neurobiology of saccadic eye movements (ed. R.H. Wurtz \& M.E. Goldberg). Elsevier Science Publisher BV (Biomedical Division).

Beswick, JA \& McCulloch, Clement 1956 Effect of hyaluronidase on the viscosity of the aqueous humour. The British journal of ophthalmology $\mathbf{4 0}$ (9), 545.

Blondeaux, P \& Vittori, G 1994 Wall imperfections as a triggering mechanism for stokeslayer transition. Journal of Fluid Mechanics 264, 107-135.

Bonfiglio, A., Lagazzo, A., Repetto, R. \& Stocchino, A. 2015 An experimental model of vitreous motion induced by eye rotations. Eye and Vision 2 (1), 10.

Bonfiglio, A., Repetto, R., Siggers, J.H. \& Stocchino, A. 2013 Investigation of the motion of a viscous fluid in the vitreous cavity induced by eye rotations and implications for drug delivery. Physics in Medicine and Biology 58 (6), 1969.

Boushehrian, H.H., Abouali, O., Jafarpur, K., Ghaffarieh, A. \& Ahmadi, G. 2016 Relationship between saccadic eye movements and formation of the krukenbergs spindlea cfd study. Mathematical medicine and biology: a journal of the IMA 34 (3), 293-312.

Brubaker, R. F. 1991 Flow of aqueous humor in humans [the friedenwald lecture]. Investigative Ophthalmology and Visual Science 32 (13), 3145-3166, PMID: 1748546.

Canning, C. R., Greaney, M. J., Dewynne, J. N. \& Fitt, A. 2002 Fluid flow in the anterior chamber of a human eye. IMA J. Math. Appl. Med. Biol. 19, 31-60.

Colombini, M. 2014 Axisymmetric flow within a torsionally oscillating sphere. Physics of Fluids 26 (2), 023602.

David, T., Smye, S., Dabbs, T. \& James, T. 1998 A model for the fluid motion of vitreous humour of the human eye during saccadic movement. Phys. Med. Biol. 43, 1385-1399.

Dvoriashyna, M., Repetto, R., Romano, M. R. \& Tweedy, J. H. 2017 Aqueous humour flow in the posterior chamber of the eye and its modifications due to pupillary block and iridotomy. Mathematical Medicine and Biology: A Journal of the IMA p. dqx012.

FitT, A. D. \& Gonzalez, G. 2006 Fluid mechanics of the human eye: Aqueous humour flow in the anterior chamber. Bull. Math. Biol. 68 (1), 53-71.

Friedland, A. B. 1978 A hydrodynamic model of acqueous flow in the posterior chamber of the eye. Bull. Math. Biol. 40, 223-235.

Helmholtz, H. 1909 Handbuch der physiologischen Optik, 3rd edn. University of Michigan Library.

Heys, J. J. \& Barocas, V. H. 2002 A boussinesq model of natural convection in the human eye and formation of krunberg's spindle. Ann. Biomed. Eng. 30, 392-401.

Jivrajka, R., Shammas, M. C., Boenzi, T., Swearingen, M. \& Shammas, H. J. 2008 Variability of axial length, anterior chamber depth, and lens thickness in the cataractous eye. Journal of Cataract \&5 Refractive Surgery 34 (2), 289-294.

Kaji, Y., Oshika, T., Usui, T. \& Sakakibara, J. 2005 Effect of shear stress on attachment of corneal endothelial cells in association with corneal endothelial cell loss after laser iridotomy. Cornea 24 (8), S55-S58.

Lide, DR, ed. 1996 CRC Handbook of Chemistry and Physics, 7rth Edn.. CRC Press Inc.

Maurice, D. M. 1998 The von sallmann lecture 1996: an ophthalmological explanation of rem sleep. Experimental eye research 66 (2), 139-145.

Meskauskas, J., Repetto, R. \& Siggers, J. H. 2011 Oscillatory motion of a viscoelastic fluid within a spherical cavity. Journal of Fluid Mechanics 685, 1-22.

Meskauskas, J., Repetto, R. \& Siggers, J. H. 2012 Shape change of the vitreous chamber influences retinal detachment and reattachment processes: Is mechanical stress during eye rotations a factor? Investigative ophthalmology $\& 3$ visual science 53 (10), 6271-6281, PMID: 22899755.

Modarreszadeh, S., Abouali, O., Ghaffarieh, A. \& Ahmadi, G. 2014 Physiology of aqueous humor dynamic in the anterior chamber due to rapid eye movement. Physiology and Behavior .

Repetto, R., Pralits, J.O., Siggers, J.H. \& Soleri, P. 2015 Phakic iris-fixated intraocular lens placement in the anterior chamber: effects on aqueous flow. Invest. Ophthalmol. Visual Sci. 56 (5), 3061-3068. 
Repetto, R., Siggers, J. H. \& Stocchino, A. 2008 Steady streaming within a periodically rotating sphere. Journal of Fluid Mechanics 608, 71-80.

Repetto, R., Siggers, J. H. \& Stocchino, A. 2010 Mathematical model of flow in the vitreous humor induced by saccadic eye rotations: effect of geometry. Biomechanics and Modeling in Mechanobiology 9 (1), 65-76.

Repetto, R., Stocchino, A. \& Cafferata, C. 2005 Experimental investigation of vitreous humour motion within a human eye model. Phys. Med. Biol. 50, 4729-4743.

Silver, D. M. \& Quigley, H. A. 2004 Aqueous flow through the iris-lens channel: estimates o the differential pressure between the anterior and the posterior chambers. J. Glaucoma 13 (2), 100-107.

Sommer, Alfred, Tielsch, JM, Katz, J, Quigley, HA, Gottsch, JD, Javitt, J \& Singh, K 1991 Relationship between intraocular pressure and primary open angle glaucoma among white and black americans. Arch ophthalmol 109 (8), 1090-1095.

TAkAhashi, K. \& Atsumi, Y. 1997 Precise measurement of individual rapid eye movements in rem sleep of humans. Sleep 20 (9), 743-752.

Tweedy, J. H., Pralits, J. O., Repetto, R. \& Soleri, P. 2017 Flow in the anterior chamber of the eye with an implanted iris-fixated artificial lens. Math. Med. Biol. p. dqx007.

Urtтi, Arto 2006 Challenges and obstacles of ocular pharmacokinetics and drug delivery. Advanced drug delivery reviews $\mathbf{5 8}$ (11), 1131-1135.

Villamarin, A., Roy, S., Hasballa, R., Vardoulis, O., Reymond, P. \& Stergiopulos, N. 2012 3D simulation of the aqueous flow in the human eye. Med. Eng. Phys. 34, 1462-1470. 